\title{
Resolvin D2 Is a Potent Endogenous Inhibitor for Transient Receptor Potential Subtype V1/A1, Inflammatory Pain, and Spinal Cord Synaptic Plasticity in Mice: Distinct Roles of Resolvin D1, D2, and E1
}

\author{
Chul-Kyu Park, ${ }^{1}$ Zhen-Zhong Xu, ${ }^{1}$ Tong Liu, ${ }^{1}$ Ning Lü, ${ }^{1}$ Charles N. Serhan, ${ }^{2}$ and Ru-Rong Ji ${ }^{1}$ \\ ${ }^{1}$ Sensory Plasticity Laboratory, Pain Research Center, and ${ }^{2}$ Center for Experimental Therapeutics and Reperfusion Injury, Harvard Institutes of Medicine, \\ Department of Anesthesiology, Perioperative and Pain Medicine, Brigham and Women's Hospital, Harvard Medical School, Boston, Massachusetts 02115
}

Inflammatory pain such as arthritic pain is typically treated with opioids and cyclo-oxygenase-2 inhibitors with well known side effects. Transient receptor potential subtype vanilloid 1 (TRPV1) and TRP ankyryn 1 (TRPA1) contribute importantly to the genesis of inflammatory pain via both peripheral mechanisms (peripheral sensitization) and spinal cord mechanisms (central sensitization). Although these TRP channels have been intensively studied, little is known about their endogenous inhibitors. Recent studies have demonstrated that the endogenous lipid mediators resolvins (RvE1 and RvD1), derived from $\omega-3$ unsaturated fatty acids, are potent inhibitors for inflammatory pain, without noticeable side effects. However, the molecular mechanisms underlying resolvins' distinct analgesic actions in mice are unclear. RvD2 is a novel family member of resolvins. Here we report that RvD2 is a remarkably potent inhibitor of TRPV1 $\left(\mathrm{IC}_{50}=0.1 \mathrm{nM}\right)$ and TRPA1 $\left(\mathrm{IC}_{50}=2 \mathrm{nM}\right)$ in primary sensory neurons, whereas RvE1 and RvD1 selectively inhibited TRPV1 $\left(\mathrm{IC}_{50}=1 \mathrm{nM}\right)$ and TRPA1 $\left(\mathrm{IC}_{50}=9 \mathrm{nM}\right)$, respectively. Accordingly, RvD2, RvE1, and RvD1 differentially regulated TRPV1 and TRPA1 agonist-elicited acute pain and spinal cord synaptic plasticity [spontaneous EPSC (sEPSC) frequency increase]. RvD2 also abolished inflammationinduced sEPSC increases (frequency and amplitude), without affecting basal synaptic transmission. Intrathecal administration of RvD2 at very low doses (0.01-1 ng) prevented formalin-induced spontaneous pain. Intrathecal RvD2 also reversed adjuvant-induced inflammatory pain without altering baseline pain and motor function. Finally, intrathecal RvD2 reversed C-fiber stimulation-evoked long-term potentiation in the spinal cord. Our findings suggest distinct roles of resolvins in regulating TRP channels and identify RvD2 as a potent endogenous inhibitor for TRPV1/TRPA1 and inflammatory pain.

\section{Introduction}

Tissue injury is known to produce inflammation and inflammatory pain. It is generally believed that inflammatory pain is driven by peripheral sensitization in primary sensory neurons following stimulation of inflammatory mediators (Hucho and Levine, 2007; Basbaum et al., 2009). Tissue injury-induced spinal cord synaptic plasticity (i.e., central sensitization) also contributes importantly to the development and maintenance of inflammatory

Received Aug. 14, 2011; revised 0ct. 11, 2011; accepted 0ct. 31, 2011.

Author contributions: C.-K.P., Z.-Z.X., T.L., N.L., and R.-R.J. designed research; C.-K.P., Z.-Z.X., T.L., and N.L. performed research; C.-K.P., Z.-Z.X., T.L., and N.L. analyzed data; R.-R.J. and C.N.S. wrote the paper.

The work was supported in part by National Institutes of Health Grants R01-DE17794 and NS54932 to R.R.J., R01-GM38765 and P01-GM095467 to C.N.S., and R01-NS67686 to both R.R.J. and C.N.S. We thank the Resolvyx Pharmaceuticals Inc. for providing resolvins and Dr. Philip J. Vickers for helpful discussion.

C.N.S. is an inventor on patents (resolvins) assigned to B.W.H. and licensed to Resolvyx Pharmaceuticals. C.N.S. is a scientific founder of Resolvyx Pharmaceuticals and owns equity in the company. C.N.S.' interests were reviewed and are managed by the Brigham and Women's Hospital and Partners HealthCare in accordance with their conflict of interest policies.

Correspondence should be addressed to Ru-Rong Ji, Department of Anesthesiology, Brigham and Women's Hospital, 75 Francis Street, Medical Research Building, Room 502, Boston, MA 02115. E-mail: rrj@@zeus. bwh.harvard.edu.

DOI:10.1523/JNEUROSCI.4192-11.2011

Copyright $\odot 2011$ the authors $\quad 0270-6474 / 11 / 3118433-06 \$ 15.00 / 0$ pain (Ji et al., 2003; Kuner, 2010). Long-term potentiation (LTP) in the spinal cord (Ruscheweyh et al., 2011) is a unique form of central sensitization for persistent pain development. Transient receptor potential vanilloid 1 (TRPV1) and ankyryn 1 (TRPA1) are two critical types of TRP channels that are strongly implicated in the genesis of inflammatory pain (Caterina et al., 2000; McMahon and Wood, 2006). Activation of TRPV1 and TRPA1 can enhance inflammatory pain not only via well demonstrated peripheral sensitization (Caterina et al., 2000; Ji et al., 2002; Bautista et al., 2006; Dai et al., 2007) but also via central sensitization, by increasing glutamate release from primary afferent terminals to enhance synaptic transmission (Kosugi et al., 2007). Great pharmaceutical effort has been devoted to developing small-molecule inhibitors of TRPV1 and TRPA1. Interestingly, several lipid mediators have been identified as endogenous activators of TRPV1 (Hwang et al., 2000; Patwardhan et al., 2009). However, little is known about the endogenous inhibitors of TRP channels.

Resolvins are endogenous lipid mediators generated during the resolution phase of acute inflammation from $\omega$-3 polyunsaturated fatty acids and display potent proresolving and antiinflammatory actions (Serhan et al., 2002, 2008). Resolvin D1 (RvD1) and RvE1 are derived from docosahexaenoic acid (DHA) 
and eicosapentaenoic acid (EPA), respectively (Serhan et al., 2008). Recent studies have shown that both peripheral (intraplantar) and central (intrathecal) administration of RvE1 and RvD1 could effectively reduce inflammatory and postoperative pain (Bang et al., 2010; Xu et al., 2010; Huang et al., 2011). Interestingly, resolvins also modulate the activity of TRP channels (Ji et al., 2011). RvE1 blocked capsaicin-induced spontaneous pain and spinal cord synaptic plasticity (Xu et al., 2010), whereas RvD1 inhibited TRPA1 in dorsal root ganglion (DRG) neurons (Bang et al., 2010). However, the efficacy ( $\mathrm{IC}_{50}$ value) of RvE1 and RvD1 in inhibiting TRPV1/TRPA1 in primary sensory neurons has not been evaluated in the previous studies.

$\mathrm{RvD2}$, derived from DHA, is a novel family member of resolvin. The complete structure of RvD2 was recently established (Spite et al., 2009). RvD2 is a potent regulator of leukocytes and controls microbial sepsis (Spite et al., 2009). Hence, the role of RvD2 in regulating TRPV1/TRPA1 and pain control was of interest. We investigated whether different resolvins play distinct roles in regulating inflammatory pain, TRPV1/TRPA1, and spinal cord synaptic plasticity.

\section{Materials and Methods}

Animals. Adult CD1 mice (male, 8-10 weeks) were used for behavioral and pharmacological studies. Young CD1 mice (4-6 weeks) were used for electrophysiological studies in spinal cord slices and DRG neurons. To produce acute and persistent inflammatory pain, diluted formalin $(5 \%, 20 \mu \mathrm{l})$, carrageenan (CRG; 1\%, $20 \mu \mathrm{l})$, or complete Freund's adjuvant (CFA; $20 \mu \mathrm{l}, 1 \mathrm{mg} / \mathrm{ml}$, Sigma) was injected into the plantar surface of a hindpaw. All the animal procedures were approved by the Animal Care Committee of Harvard Medical School.

Drugs and administration. Capsaicin, carrageenan, allyl isothiocyanate (AITC), and guanosine $5^{\prime}$ - $[\beta$-thio] diphosphate trilithium salt (GDP $\beta S$ ) were obtained from Sigma. AMG 9810 [(2E)- $N$-(2,3-dihydro-1,4benzodioxin-6-yl)-3-[4-(1,1-dimethylethyl)phenyl]-2-propenamide] and pertussis toxin were purchased from Tocris Bioscience. RvD1, RvD2, DHA, and EPA were purchased from Cayman Chemical. RvE1 was a kind gift from Resolvyx Pharmaceuticals. Resolvins were initially isolated in exudates from the resolution phase of self-limited acute inflammation (Serhan et al., 2002). After the full structural elucidation, resolvin's physical and anti-inflammatory properties were confirmed by total organic synthesis (Serhan et al., 2008). All resolvins were prepared in PBS. For intrathecal injection, spinal cord puncture was made with a 30 gauge needle between the L5 and L6 level to deliver reagents ( $10 \mu \mathrm{l})$ to the CSF.

Whole-cell patch-clamp recordings in cultured DRG neurons. DRG neuron cultures were prepared as previously reported (Park et al., 2011). DRG cells were plated on glass coverslips and cultured in a neurobasal defined medium (with 2\% B27 supplement, Invitrogen) for $24 \mathrm{~h}$ before use. Whole-cell voltage-clamp recordings were performed at room temperature $\left(28^{\circ} \mathrm{C}\right)$ to measure inward currents with Axopatch-200B amplifier (Molecular Devices). The pipette solution for voltage-clamp contains the following (in mM): $126 \mathrm{~K}$-gluconate, $10 \mathrm{NaCl}, 1 \mathrm{MgCl}_{2}, 10$ EGTA, 2 $\mathrm{NaATP}$, and $0.1 \mathrm{MgGTP}$, adjusted to $\mathrm{pH} 7.4$ (with $\mathrm{KOH}$ ) and osmolarity 295-300 mOsm. In some cases, GDP $\beta S(2.5 \mathrm{~mm})$ was included in intracellular solution to block GPCRs. Extracellular solution was prepared by adding $0 \mathrm{~mm} \mathrm{CaCl}_{2}$ and $2 \mathrm{~mm}$ EGTA for $\mathrm{Ca}^{2+}$ chelation. It contains the following (in $\mathrm{mm}$ ): $140 \mathrm{NaCl}, 5 \mathrm{KCl}, 2 \mathrm{CaCl}_{2}, 1 \mathrm{MgCl}_{2}, 10$ HEPES, 10 glucose, adjusted to $\mathrm{pH}$ 7.4. The recording chamber $(300 \mu \mathrm{l})$ was continuously superfused $(2-3 \mathrm{ml} / \mathrm{min})$. Series resistance was compensated for $(>80 \%)$, and leak subtraction was performed. Data were low-pass filtered at $2 \mathrm{kHz}$, sampled at $10 \mathrm{kHz}$. The pClamp8 (Molecular Devices) software was used during experiments and analysis. Voltage-clamp was performed at a holding potential of $-60 \mathrm{mV}$.

Spinal cord slice preparation and patch-clamp recordings. A portion of the lumbar spinal cord (L4-L5) was removed from mice, as previously reported (Xu et al., 2010). Transverse slices $(400-600 \mu \mathrm{m})$ were cut on a vibrating microslicer. The slices were perfused with Kreb's solution $(8-10 \mathrm{ml} / \mathrm{min})$ saturated with $95 \% \mathrm{O}_{2}$ and $5 \% \mathrm{CO}_{2}$ at $37^{\circ} \mathrm{C}$. The whole- cell patch-clamp recordings were made from lamina IIo neurons in voltage-clamp mode at $37^{\circ} \mathrm{C}$. After establishing the whole-cell configuration, neurons were held at the potential of $-70 \mathrm{mV}$ to record sEPSCs. The internal solution contains the following (in mM): $135 \mathrm{~K}$-gluconate, 5 $\mathrm{KCl}, 0.5 \mathrm{CaCl}$, $2 \mathrm{MgCl}$, 5 EGTA, 5 HEPES, and 5 ATP-Mg, adjusted to $\mathrm{pH}$ 7.4. The perfusion solution contains the following (in $\mathrm{mm}$ ): $117 \mathrm{NaCl}$, $3.6 \mathrm{KCl}, 2.5 \mathrm{CaCl}_{2}, 1.2 \mathrm{MgCl}_{2}, 1.2 \mathrm{NaH}_{2} \mathrm{PO}_{4}, 25 \mathrm{NaHCO}_{3}$, and 11 glucose. The resistance of a typical patch pipette is $5-10 \mathrm{M} \Omega$. Membrane currents were amplified with an Axopatch 200B amplifier (Molecular Devices) in voltage-clamp mode. Signals were filtered at $2 \mathrm{kHz}$ and digitized at $5 \mathrm{kHz}$. Data were stored with a personal computer using pCLAMP 10 software and analyzed with Mini Analysis (Synaptosoft).

Spinal cord LTP recordings in anesthetized mice. Spinal cord LTP was recorded in vivo as we recently demonstrated (Park et al., 2011). Mice were anesthetized with urethane $(1.5 \mathrm{~g} / \mathrm{kg}$, i.p.). A laminectomy was performed at vertebrae T13-L1 to expose the lumbar enlargement, and the left sciatic nerve was exposed for bipolar electrical stimulation. The vertebral column was firmly suspended by rostral and caudal clamps on the stereotaxic frame. Following electrical stimulation of the sciatic nerve, the field potentials were recorded in the ipsilateral L4-L5 spinal cord segments with glass microelectrodes, $100-300 \mu \mathrm{m}$ from the surface of the cord. After recording stable responses following test stimuli $(2 \times$ C-fiber threshold, $0.5 \mathrm{~ms}, 1 \mathrm{~min}$ interval, every $5 \mathrm{~min}$ ) for $>40 \mathrm{~min}$, conditioning tetanic stimulation $(5 \times \mathrm{C}$-fiber threshold, $100 \mathrm{~Hz}, 1 \mathrm{~s}$, four trains, $10 \mathrm{~s}$ interval) was delivered to the sciatic nerve for inducing LTP of C-fiber-evoked field potentials. For intrathecal drug delivery, a PE5 catheter was inserted at the L5-L6 level via lumbar puncture.

Behavioral analysis. Animals were habituated to the testing environment for $2 \mathrm{~d}$ and were kept in plastic boxes for $30 \mathrm{~min}$ of habituation before testing. Heat sensitivity was tested by radiant heat using a Hargreaves apparatus (Hargreaves et al., 1988). For testing capsaicin and mustard oil AITC-induced spontaneous pain (nocifensive behavior), capsaicin or AITC was intraplantarly injected, and the time spent on nocifensive behavior (flinching and licking) was recorded for $5 \mathrm{~min}$. For the formalin test, the time spent in licking and flinching the affected paws was recorded every $5 \mathrm{~min}$ for $45 \mathrm{~min}$. For testing mechanical sensitivity, mice were put in boxes on an elevated metal mesh floor, and the plantar surface of each hindpaw was stimulated with a series of von Frey hairs with logarithmically incrementing stiffness $(0.02-2.56 \mathrm{~g}$, Stoelting), presented perpendicular to the plantar surface. The $50 \%$ paw withdrawal threshold was determined using Dixon's up-down method. For the rotarod test, mice were put on the treadmill with accelerating speed, and the seconds spent before the first fall were recorded. For all the behavioral tests, the experimenters were blinded to the treatments.

Statistical analysis. All data were expressed as mean \pm SEM. Differences between groups were compared using Student's $t$ test (two groups), one-way ANOVA (multiple groups) followed by post hoc Bonnferroni's test, or two-way ANOVA for analyzing the LTP data. The criterion for statistical significance was $p<0.05$.

\section{Results}

\section{RvD2, RvE1, and RvD1 differentially inhibit TRPV1 and TRPA1 currents in dissociated DRG neurons}

First, we used patch-clamp recordings in dissociated mouse DRG neurons to test whether RvD2 would modulate TRPV1 and TRPA1 activity. Perfusion of DRG neurons with capsaicin (100 nM) elicited a marked TRPV1 current, and this current was dose-dependently inhibited by RvD2 (Fig. $1 A, B$ ). Strikingly, RvD2 inhibited TRPV1 currents with an extremely low $\mathrm{IC}_{50}, 0.1 \mathrm{~nm}$ (i.e., $0.04 \mathrm{ng} / \mathrm{ml}$ ) (Fig. 1 B; Table 1). For comparison, we also tested the efficacy of RvE1 and RvD1 in inhibiting TRPV1 current. Of interest, RvE1 inhibited TRPV1 currents with $\mathrm{IC}_{50}=1.0 \mathrm{nM}$, which is 10 times higher than that of RvD2, whereas RvD1 had no effect even at high concentrations (Fig. 1C; Table 1).

Next, we examined whether RvD2 would also modulate TRPA1 current, induced by mustard oil AITC (300 $\mu \mathrm{M})$. RvD2 
A

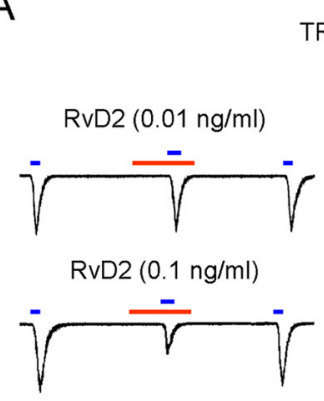

TRPV1 current
B

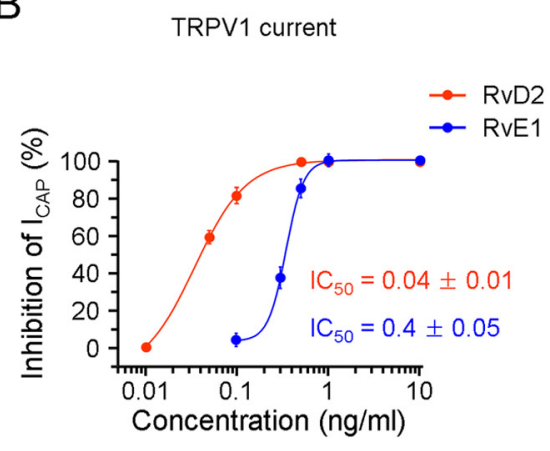

E

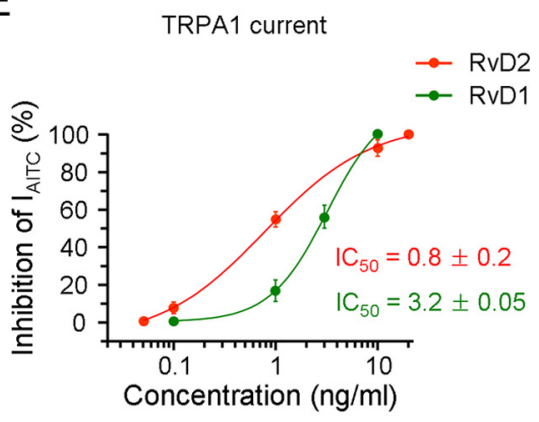

C

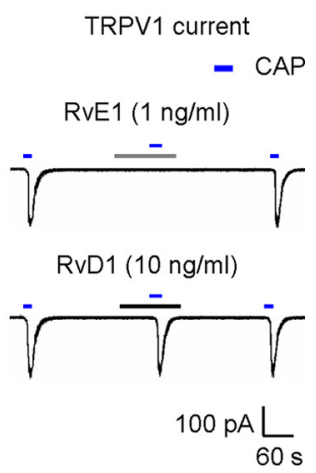

F

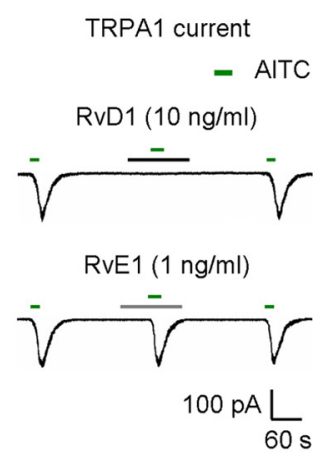

G

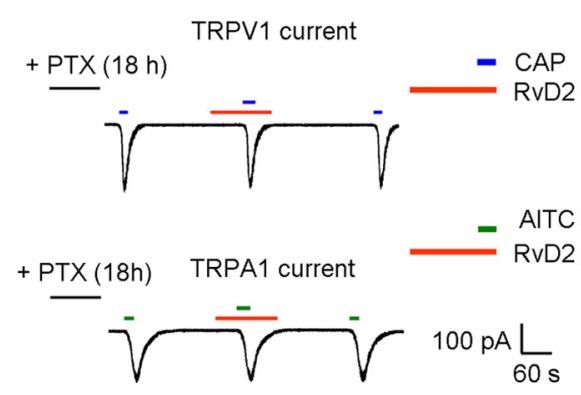

$\mathrm{H}$

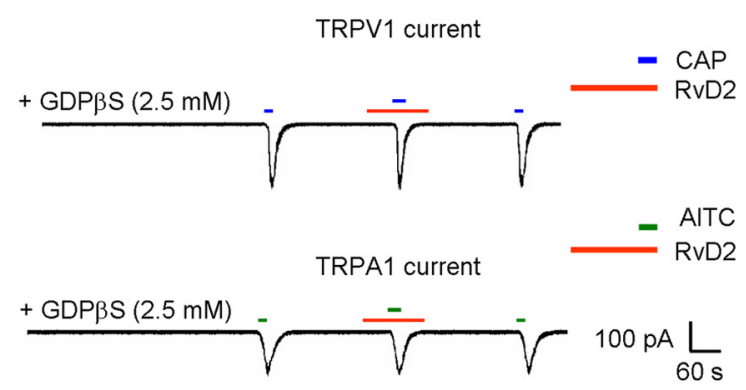

Figure 1. RvD2, RvE1, and RvD1 differentially regulate TRPV1 and TRPA1 currents in DRG neurons via GPCRs. $A$, Capsaicin (CAP; 100 nM)-induced inward currents. Note a dose-dependent inhibition of TRPV1 currents by RvD2. B, Dose-response curves showing inhibition of TRPV1 currents by RvD2 and RvE1. IC ${ }_{50}$ of TRPV1 current inhibition is calculated and indicated inside the graph. C, Inhibition of TRPV1 current by RvE1 but not RvD1. D, Mustard oil AITC ( $300 \mu \mathrm{m})$-induced inward currents. Note a dose-dependent inhibition of TRPA1 currents by RvD2. E, Dose-response curves showing inhibition of TRPA1 currents by RvD2 and RvD1. IC $C_{50}$ of TRPA1 current inhibition is calculated and indicated inside the graph. $\boldsymbol{F}$, Inhibition of TRPA1 current by RvD1 but not RvE1. $\boldsymbol{G}$, Pretreatment of DRG cultures with PTX $(0.5 \mu \mathrm{g} / \mathrm{ml}, 18 \mathrm{~h})$ blocks the inhibitory effects of RvD2 $(1 \mathrm{ng} / \mathrm{ml})$ on TRPV1 current (top) and TRPA1 current (bottom). $\boldsymbol{H}$, Intracellular perfusion of GDP $\beta S$ ( 2.5 $\mathrm{mm}, 8 \mathrm{~min}$ ) blocks the inhibitory effects of RvD2 (1 ng/ml) on TRPV1 current (top) and TRPA1 current (bottom). $n=5-8$ neurons. All results are means \pm SEM.

Table 1. IC 5 for inhibition of TRPV1 and TRPA1 currents by RvD2, RvD1, RvE1, and their fatty acid precursors in DRG neurons

\begin{tabular}{llcc}
\hline Inhibitors & Molecular weight & TRPV1 I $_{50}(\mathrm{~nm})$ & TRPA1 I $_{50}(\mathrm{nM})$ \\
\hline RvE1 & 350.4 & $1.0 \pm 0.1$ & $>28.5$ \\
RvD1 & 376.5 & $>26.6$ & $8.5 \pm 0.1$ \\
RvD2 & 376.5 & $0.1 \pm 0.01$ & $2.1 \pm 0.5$ \\
DHA & 328.5 & $1200.0 \pm 20.0$ & $>304,000.0$ \\
EPA & 302.5 & $224.0 \pm 10.0$ & $>330,578.0$ \\
\hline
\end{tabular}

TRPV1 and TRPA1 currents were induced by capsaicin $(100 \mathrm{nM})$ and AITC $(300 \mu \mathrm{M})$, respectively.

potently inhibited TRPA1 currents with $\mathrm{IC}_{50}=2.1 \mathrm{~nm}$ (Fig. $1 D, E$; Table 1). In sharp contrast, RvD1 inhibited TRPA1 currents with $\mathrm{IC}_{50}=8.5 \mathrm{nM}$, which is 4 times higher than that of RvD2, whereas RvE1 did not inhibit TRPA1 currents even at high concentrations (Fig. 1F; Table 1).

Notably, the molecular weights of RvE1 (350.4), RvD1 (376.5), and RvD2 (376.5), as well as their precursors DHA
(328.5) and EPA (302.5) are comparable (Table 1). Further comparison revealed that DHA and EPA inhibited TRPV1 currents with $\mathrm{IC}_{50}$ values of 1200 and $224 \mathrm{nM}$, respectively, without affecting TRPA1 currents (Table 1). In addition, AMG 9810, a commonly used TRPV1 antagonist, inhibited TRPV1 currents with $\mathrm{IC}_{50}=163 \mathrm{~nm}$ (Park et al., 2011), which is $\approx 1600$ times higher than that of RvD2. Collectively, these results indicate that resolvins and their precursors differentially regulate TRPV1 and TRPA1 currents.

Proresolving mediators such as lipoxin A4, RvE1, and RvD1 each signal via specific GPCRs (Serhan et al., 2008). To determine the involvement of GPCRs in resolvins' actions, we pretreated DRG cultures with a selective G $\alpha$ i-coupled GPCR inhibitor, pertussis toxin (PTX; $0.5 \mu \mathrm{g} / \mathrm{ml}$ ) for $18 \mathrm{~h}$. After PTX treatment, RvD2 could not alter TRPV1 and TRPA1 currents (Fig. 1G) $(p>$ $0.05, n=6$ neurons). We also blocked the action of G-proteins by intracellular delivery of GDP $\beta S(2.5 \mathrm{~mm}, 8 \mathrm{~min})$ via recording 
A

Capsaicin and mustard oil-induced pain

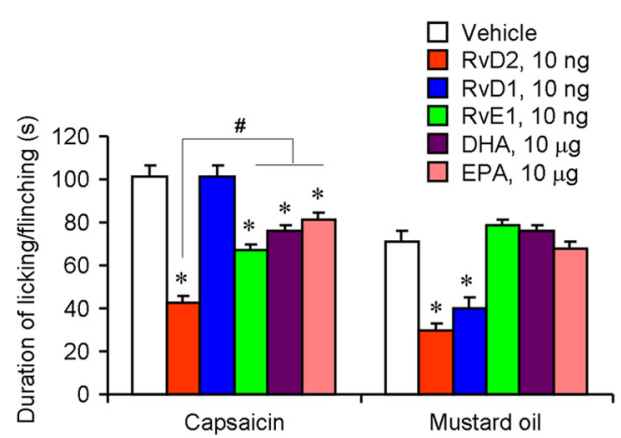

B

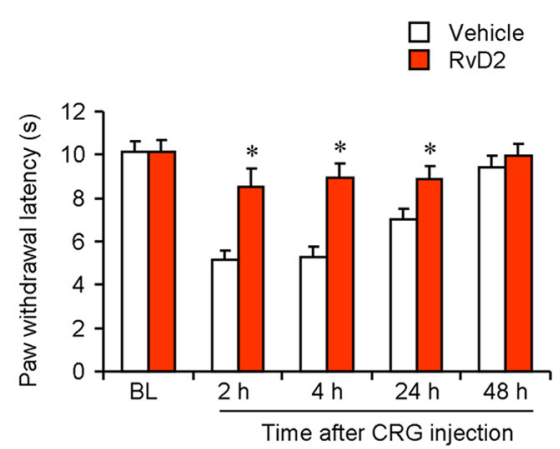

C

CRG-induced mechanical allodynia

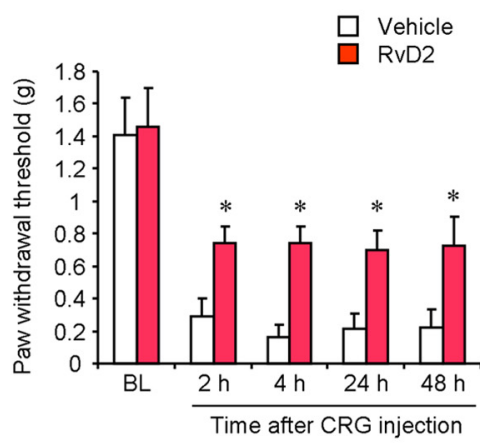

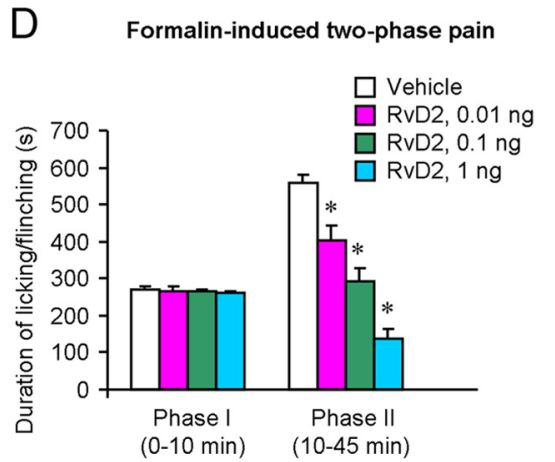

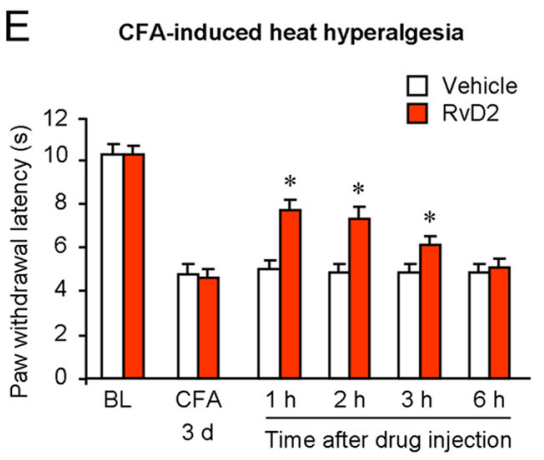

$\mathrm{F}$

Baseline pain

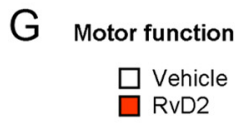

Figure 2. Intraplantar and intrathecal administration of RvD2 inhibits inflammatory pain. $A$, Intraplantar administration of RvD2, RvD1, RvE1, DHA, or EPA reduces capsaicin-induced (1 $\mu \mathrm{g})$ and AlTC-induced (1 $\mu \mathrm{g}$ ) spontaneous pain. ${ }^{*} p<0.05$, versus vehicle; ${ }^{*} p<0.05 . \boldsymbol{B}, \boldsymbol{C}$, CRG-induced heat hyperalgesia $(\boldsymbol{B})$ and mechanical allodynia $(\boldsymbol{C})$ in vehicle-treated and RvD2-treated (10 ng, intraplantar) mice. ${ }^{*} p<0.05$, versus vehicle. $\boldsymbol{D}$, Formalin-induced first- and second-phase pain in vehicle- and RvD1-treated (0.01-1 ng, i.t.) mice. ${ }^{*} p<0.05$, versus vehicle. $\boldsymbol{E}$, CFA-induced heat hyperalgesia on day 3 and its reduction by RvD2 posttreatment $\left(10 \mathrm{ng}\right.$, i.t.). ${ }^{*} p<0.05$, versus vehicle. $\boldsymbol{F}, \boldsymbol{G}, \operatorname{RvD2}(10 \mathrm{ng}$, i.t.) does not alter baseline heat sensitivity $(\boldsymbol{F})$ and motor function $(\boldsymbol{G}$, rota-rod test) in naive animals. $n=5-8$ mice. All results are means \pm SEM.

electrode. GDP $\beta$ S treatment completely blocked RvD2's inhibition of TRPV1 and TRPA1 currents (Fig. $1 H$ ). Together, these data suggest that (1) RvD2 does not act directly on TRPV1/ TRPA1 and (2) RvD2's effects are mediated by specific G $\alpha$ icoupled GPCRs in DRG neurons.

\section{RvD2, RvE1, and RvD1 differentially inhibit TRPV1- and TRPA1-induced spontaneous pain}

We further examined whether specific resolvins could differentially regulate TRPV1- and TRPA1-mediated pain in mice. Intraplantar injection of capsaicin $(1 \mu \mathrm{g})$ or AITC $(1 \mu \mathrm{g})$ elicited marked spontaneous pain behaviors (flinching/licking) in mice. Both capsaicin- and AITC-induced nocifensive behaviors were significantly reduced by intraplantar RvD2 (10 ng) (Fig. 2A). In contrast, intraplantar RvE1 and RvD1 (10 ng) each selectively inhibited capsaicin- and AITC-induced pain. At much higher doses $(10 \mu \mathrm{g})$, the precursors EPA and DHA also inhibited capsaicin-induced pain, but not AITC-induced pain. Of note, RvD2 was more potent than RvE1 in inhibiting capsaicininduced spontaneous pain (Fig. 2A). Therefore, both electrophysiological and behavioral data suggest distinct roles of resolvins in modulating TRPV1/TRPA1-elicited inward currents and pain behaviors.

\section{Peripheral and central administration of RvD2 potently} inhibits inflammatory pain

Intraplantar injection of formalin, CRG, and CFA is known to elicit acute, subacute, and chronic inflammatory pain, respectively (Xu et al., 2010). Peripheral (intraplantar) administration of RvD2 (10 ng) largely prevented CRG-induced heat hyperalgesia (Fig. 2 B) and me- chanical allodynia (Fig. 2C), and reduced CRG-induced paw edema (data not shown). Spinal (intrathecal) pre-emptive treatment of RvD2 (0.01-1 ng) also dose-dependently inhibited formalininduced spontaneous pain in the second phase but not in the first phase (Fig. 2D), indicating a specific inhibition of central sensitization. Strikingly, even $0.01 \mathrm{ng}$ of RvD2 had significant antinociceptive action (Fig. 2D).

In addition to preventing the development of inflammatory pain, RvD2 also inhibited persistent inflammatory pain after post-treatment. Intrathecal administration of $\operatorname{RvD} 2(10 \mathrm{ng}), 3 \mathrm{~d}$ after CFA-induced inflammation, effectively reduced CFAinduced heat hyperalgesia and mechanical allodynia for $>3 \mathrm{~h}$ (Fig. 2E; data not shown).

We further tested the effects of RvD2 on baseline pain and motor function. RvD2 (10 ng, i.t.) did not alter baseline pain sensitivity to thermal and mechanical stimulation (Fig. 2 F; data not shown). Neither did this treatment affect the animal's motor function in the rota-rod test (Fig. 2G). Thus, RvD2 can potently attenuate inflammatory pain in acute, subacute, and persistent/ chronic phase, without affecting normal pain perception and motor function.

\section{RvD2, RvE1, and RvD1 differentially inhibit TRPV1- and TRPA1-induced spinal cord synaptic plasticity}

Given an important role of synaptic plasticity in generating inflammatory pain (Ji et al., 2003), we assessed the different roles of RvD2, RvE1, and RvD2 in spinal cord synaptic plasticity induced by TRPV1/TRPA1 agonist. It is generally believed that changes in the frequency and amplitude of spontaneous EPSCs (sEPSCs) in spinal cord neurons are caused by altered glutamate neurotrans- 
A

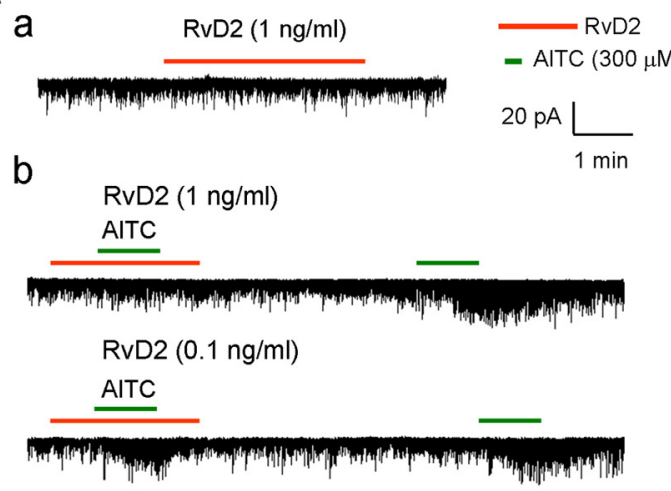

C

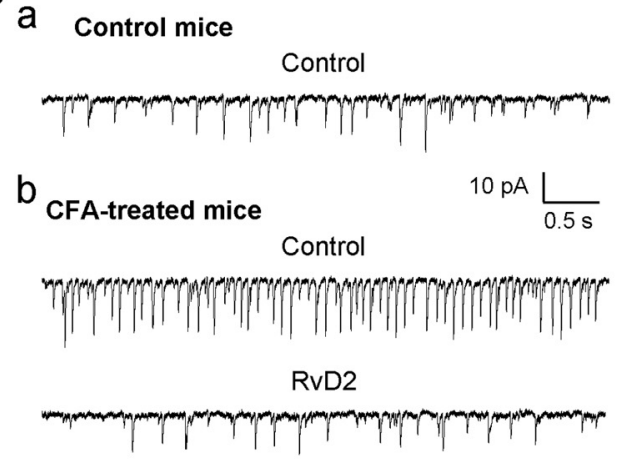

B

sEPSC Frequency

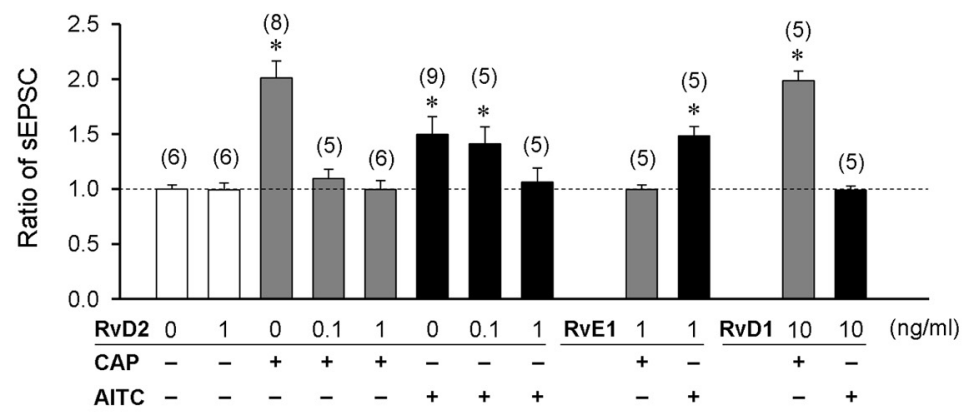

$E$

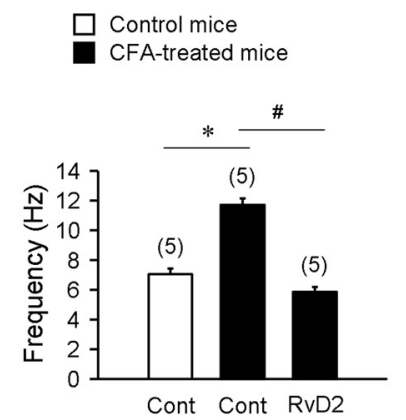

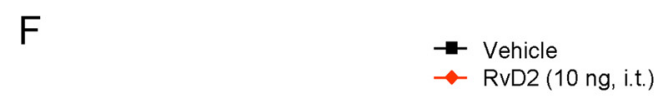

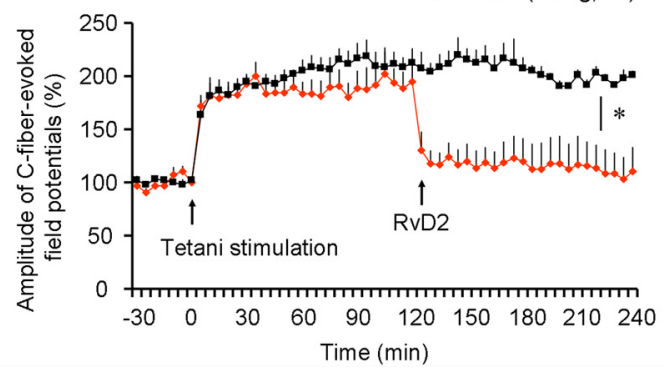

Figure 3. RvD2 abolishes spinal cord synaptic plasticity and reverses spinal cord LTP. A-E, sEPSCs in lamina II neurons of spinal cord slices. Aa, Traces of sEPSCs showing no effect of RvD2 on baseline sEPSCs. $\boldsymbol{A} \boldsymbol{b}$, Traces of SEPSCs showing dose-dependent inhibition of AITC-induced enhancement of sEPSCs by RvD2 $(0.1 \mathrm{and} 1 \mathrm{ng} / \mathrm{ml})$. $\boldsymbol{B}$, Ratio of sEPSC frequency after treatment of capsaicin (CAP; $0.1 \mu \mathrm{m})$, AITC ( $300 \mu \mathrm{M}), \operatorname{RvD2}(0.1 \mathrm{and} 1 \mathrm{ng} / \mathrm{ml})$, RvE1 $(1 \mathrm{ng} / \mathrm{ml})$, and RvD1 $(10 \mathrm{ng} / \mathrm{ml}) .{ }^{*} p<0.05$, versus pretreatment baseline. $n=5 \sim 9$ neurons. Note that capsaicin and AITC increase the frequency of sEPSC, which is differentially blocked by RvD2, RvE1, and RvD1. C, Traces of sEPSC in a spinal cord slice from control (a) and CFA-inflamed (1 d; $\boldsymbol{b})$ mice showing sEPSC increase after inflammation and its inhibition by RvD2 (1 ng/ml). D, E, sEPSC frequency (D) and amplitude $(\boldsymbol{E})$ in the control and CFA-inflamed conditions, and the actions of RvD2. ${ }^{*} p<0.05$, compared with noninflamed control; $" \# p 0.05$, versus CFA-inflamed control. $n=5$ neurons. Cont, Control. The number of neurons recorded is indicated on the top of each column. $\boldsymbol{F}$, Reversal of LTP of C-fiber-evoked field potentials in the dorsal horn of anesthetized mice by RvD2 (10 ng, i.t.), administered $2 \mathrm{~h}$ after LTP induction. ${ }^{*} p<0.05$ (vehicle vs RvD2, two-way ANOVA, $n=5$ mice). All results are means \pm SEM.

mission via respective presynaptic and postsynaptic mechanisms (Xu et al., 2010). Patch-clamp recording in lamina II neurons of spinal cord slices showed that RvD2 alone $(1 \mathrm{ng} / \mathrm{ml})$ altered neither the sEPSC frequency (Fig. 3A,B) nor sEPSC amplitude ( $p>0.05, n=5$ neurons). Capsaicin $(1 \mu \mathrm{M})$ and AITC (300 $\mu \mathrm{M})$ substantially increased the frequency of sEPSCs (Fig. $3 A, B)$ but not the amplitude of sEPSCs $(p>0.05, n=6)$, indicating that TRPV1/TRPA1 agonist potentiated glutamate release from presynaptic terminals of primary afferents. Notably, the capsaicin- and AITC-induced increases in sEPSC frequency were dose-dependently blocked by RvD2 (0.1 and $1 \mathrm{ng} /$ $\mathrm{ml}$, respectively) (Fig. $3 A, B$ ). Instead, RvE1 and RvD1 only selectively inhibited capsaicin- and AITC-induced sEPSC frequency increase, respectively (Fig. $3 A, B$ ). Notably, the concen- trations required for inhibiting TRPV1/TRPA1-evoked synaptic transmission are as follows: RvD2 $<\mathrm{RvE} 1<\mathrm{RvD} 1$.

\section{RvD2 blocks inflammation-induced synaptic plasticity and reverses LTP in the spinal cord}

CFA elicited significant increases both in sEPSC frequency (68\%) and amplitude (23\%) in spinal cord slices prepared from inflamed mice ( $1 \mathrm{~d})$. Strikingly, these increases in sEPSC frequency and amplitude were abolished by RvD2 at a low concentration ( 1 $\mathrm{ng} / \mathrm{ml}$ ) (Fig. 3C-E), indicating that RvD2 could modulate synaptic plasticity via both presynaptic (sEPSC frequency) and postsynaptic (sEPSC amplitude) mechanisms.

C-fiber-induced spinal LTP is a persistent form of synaptic plasticity in the spinal cord (Ruscheweyh et al., 2011). Tetanic 
stimulation of the C-fibers in the sciatic nerve evoked marked LTP of C-fiber-evoked field potentials in the dorsal horn (Fig. $3 F$ ), which was completely reversed by RvD2 (10 ng), intrathecally administrated $2 \mathrm{~h}$ after LTP induction (Fig. $3 F)(p<0.05$, two-way ANOVA).

\section{Discussion}

Although great effort in pain research and drug development has been devoted to developing TRPV1 and TRPA1 antagonists, little is known about the endogenous inhibitors of TRPV1/TRPA1. Resolvins are endogenous anti-inflammatory and proresolving lipid mediators produced during the resolution phase of inflammation (Serhan et al., 2008). The present study has identified resolvins as highly potent endogenous inhibitors for TRPV1/ TRPA1. In particular, we demonstrated distinct roles of RvD1, RvD2, and RvE1 in regulating TRPV1 and TRPA1 activity. Although previous studies indicated different inhibition of TRPV1 and TRPA1 by RvE1 (Xu et al., 2010) and RvD1 (Bang et al., 2010), respectively, the present study further calculated the $\mathrm{IC}_{50}$ for RvE1 inhibition of TRPV1 $\left(\mathrm{IC}_{50}=1.0 \mathrm{nM}\right)$ and RvD1 inhibition of TRPA $1\left(\mathrm{IC}_{50}=8.5 \mathrm{nM}\right)$. The most striking finding of this study is that RvD2, a novel member of the resolvin family, inhibited both TRPV1 and TRPA1 with superior potency: $\mathrm{IC}_{50}=0.1$ nM for TRPV1 and $\mathrm{IC}_{50}=2.1 \mathrm{~nm}$ for TRPA1. To the best of our knowledge, RvD2 is probably the most potent inhibitor of TRPV1, among all the published compounds. Importantly, RvD2's inhibitory actions are mediated by specific GPCRs, which can clearly separate this endogenous inhibitor from classic TRPV1/TRPA1 antagonists that act directly on the channels. In parallel, RvD2 also inhibited TRPV1/TRPA1-induced spontaneous pain, as well as TRPV1/TRPA1 and inflammation-evoked spinal cord synaptic plasticity (sEPSC increases).

Our findings demonstrated that peripheral and central administration of RvD2, at very low doses (0.01-10 ng), reduced inflammatory pain in acute, subacute, and persistent conditions. RvD2 also reduced different modalities of inflammatory pain including spontaneous pain and evoked pain (heat hyperalgesia and mechanical allodynia), as a result of inhibiting TRPV1/ TRPA1 and inflammation-induced synaptic plasticity. In addition, RvD2 may also attenuate inflammatory pain via TRPV1/ TRPA1-independent mechanisms. For example, RvD2 but not the TRPV1 antagonist AMG 9810 reversed spinal cord LTP (Fig. 3F) (Park et al., 2011).

Inflammatory pain such as arthritic pain is typically treated with opioids and cyclo-oxygenase-2 inhibitors with well known side effects (Sommer and Birklein, 2010; Ji et al., 2011). Given its high potency and wide safety range, RvD2 and its related compounds are promising new therapeutics for treating pain associated with inflammation. However, the detailed signaling mechanisms of $\mathrm{RvD} 2$ in pain relief remain unclear. It is important to understand these molecular means that endow RvD2 to be so potent for pain relief. Elucidation of the RvD2 signaling mechanisms is also critical in future clinical development for targeting the $\mathrm{RvD} 2$ receptors and signaling pathways with small-molecule agonists.

\section{References}

Bang S, Yoo S, Yang TJ, Cho H, Kim YG, Hwang SW (2010) Resolvin D1 attenuates activation of sensory transient receptor potential channels leading to multiple antinociception. Br J Pharmacol 161:707-720.
Basbaum AI, Bautista DM, Scherrer G, Julius D (2009) Cellular and molecular mechanisms of pain. Cell 139:267-284.

Bautista DM, Jordt SE, Nikai T, Tsuruda PR, Read AJ, Poblete J, Yamoah EN, Basbaum AI, Julius D (2006) TRPA1 mediates the inflammatory actions of environmental irritants and proalgesic agents. Cell 124:1269-1282.

Caterina MJ, Leffler A, Malmberg AB, Martin WJ, Trafton J, Petersen-Zeitz KR, Koltzenburg M, Basbaum AI, Julius D (2000) Impaired nociception and pain sensation in mice lacking the capsaicin receptor. Science 288:306-313.

Dai Y, Wang S, Tominaga M, Yamamoto S, Fukuoka T, Higashi T, Kobayashi K, Obata K, Yamanaka H, Noguchi K (2007) Sensitization of TRPA1 by PAR2 contributes to the sensation of inflammatory pain. J Clin Invest 117:1979-1987.

Hargreaves K, Dubner R, Brown F, Flores C, Joris J (1988) A new and sensitive method for measuring thermal nociception in cutaneous hyperalgesia. Pain 32:77-88.

Huang L, Wang CF, Serhan CN, Strichartz G (2011) Enduring prevention and transient reduction of postoperative pain by intrathecal resolvin D1. Pain 152:557-565.

Hucho T, Levine JD (2007) Signaling pathways in sensitization: toward a nociceptor cell biology. Neuron 55:365-376.

Hwang SW, Cho H, Kwak J, Lee SY, Kang CJ, Jung J, Cho S, Min KH, Suh YG, Kim D, Oh U (2000) Direct activation of capsaicin receptors by products of lipoxygenases: endogenous capsaicin-like substances. Proc Natl Acad Sci U S A 97:6155-6160.

Ji RR, Samad TA, Jin SX, Schmoll R, Woolf CJ (2002) p38 MAPK activation by NGF in primary sensory neurons after inflammation increases TRPV1 levels and maintains heat hyperalgesia. Neuron 36:57-68.

Ji RR, Kohno T, Moore KA, Woolf CJ (2003) Central sensitization and LTP: do pain and memory share similar mechanisms? Trends Neurosci 26:696-705.

Ji RR, Xu ZZ, Strichartz G, Serhan CN (2011) Emerging roles of resolvins in the resolution of inflammation and pain. Trends Neurosci 34:599-609.

Kosugi M, Nakatsuka T, Fujita T, Kuroda Y, Kumamoto E (2007) Activation of TRPA 1 channel facilitates excitatory synaptic transmission in substantia gelatinosa neurons of the adult rat spinal cord. J Neurosci 27:4443-4451.

Kuner R (2010) Central mechanisms of pathological pain. Nat Med $16: 1258-1266$.

McMahon SB, Wood JN (2006) Increasingly irritable and close to tears: TRPA1 in inflammatory pain. Cell 124:1123-1125.

Park CK, Lü N, Xu ZZ, Liu T, Serhan CN, Ji RR (2011) Resolving TRPV1and TNF- $\alpha$-mediated spinal cord synaptic plasticity and inflammatory pain with neuroprotectin D1. J Neurosci 31:15072-15085.

Patwardhan AM, Scotland PE, Akopian AN, Hargreaves KM (2009) Activation of TRPV1 in the spinal cord by oxidized linoleic acid metabolites contributes to inflammatory hyperalgesia. Proc Natl Acad Sci U S A 106:18820-18824.

Ruscheweyh R, Wilder-Smith O, Drdla R, Liu XG, Sandkühler J (2011) Long-term potentiation in spinal nociceptive pathways as a novel target for pain therapy. Mol Pain 7:20.

Serhan CN, Hong S, Gronert K, Colgan SP, Devchand PR, Mirick G, Moussignac RL (2002) Resolvins: a family of bioactive products of omega-3 fatty acid transformation circuits initiated by aspirin treatment that counter proinflammation signals. J Exp Med 196:1025-1037.

Serhan CN, Chiang N, Van Dyke TE (2008) Resolving inflammation: dual anti-inflammatory and pro-resolution lipid mediators. Nat Rev Immunol $8: 349-361$

Sommer C, Birklein F (2010) Fighting off pain with resolvins. Nat Med 16:518-520.

Spite M, Norling LV, Summers L, Yang R, Cooper D, Petasis NA, Flower RJ, Perretti M, Serhan CN (2009) Resolvin D2 is a potent regulator of leukocytes and controls microbial sepsis. Nature 461:1287-1291.

Xu ZZ, Zhang L, Liu T, Park JY, Berta T, Yang R, Serhan CN, Ji RR (2010) Resolvins RvE1 and RvD1 attenuate inflammatory pain via central and peripheral actions. Nat Med 16:592-597. 